\title{
Elastic Registration of 3D Ultrasound Images
}

\author{
Pezhman Foroughi ${ }^{1}$ and Purang Abolmaesumi ${ }^{1,2}$ \\ 1 Department of Electrical and Computer Engineering, Queen's University, Canada \\ 2 School of Computing, Queen's University, Canada \\ purang@cs.queensu.ca
}

\begin{abstract}
D registration of ultrasound images is an important and fast-growing research area with various medical applications, such as image-guided radiotherapy and surgery. However, this registration process is extremely challenging due to the deformation of soft tissue and the existence of speckles in these images. This paper presents a novel intra-modality elastic registration technique for 3D ultrasound images. It uses the general concept of attribute vectors to find the corresponding voxels in the fixed and moving images. The method does not require any pre-segmentation and does not employ any numerical optimization procedure. Therefore, the computational requirements are very low and it has the potential to be used for real-time applications. The technique is implemented and tested for 3D ultrasound images of liver, captured by a 3D ultrasound transducer. The results show that the method is sufficiently accurate and robust and does not easily get trapped with local minima.
\end{abstract}

\section{Introduction}

Ultrasound is an easy-to-use, inexpensive and real-time imaging tool with no ionizing radiation which is widely used for diagnosis applications. Recently, there has been major interest in the employment of this imaging technique for intraoperative registration in radiotherapy and image-guided surgery. Furthermore, ultrasound registration could be used for measuring tissue mechanics properties. However, due to speckles and shadowing effects, the registration of this imaging modality is a challenging process. Previous attempts include the development of techniques for registration of ultrasound to the other image modalities such as CT and MRI [1-5], as well as intra-modality registration of these images [6-9].

Several methods have been used for elastic registration of ultrasound images. Shekhar et al. 6] investigated the registration of ultrasound volumes based on the mutual information measure. Median filter was found to be the proper smoothing filter for this method. The results of different transformation modes were presented. Krucker et al. 7 developed a subvolume-based algorithm for elastic ultrasound registration. The image was divided into subvolumes interactively and local rigid registrations were computed. Connectivity of the entire volume was ensured by global interpolation using thin-plate splines after each iteration. Xiao et al. 8 used a correlation-based approach for registration of 3D free-hand ultrasound images of the breast. Deformable registration of cardiac images has been investigated more specifically in [9, 10. 
In this paper, we propose a novel technique for 3D elastic registration of ultrasound images. In our approach, the concept of attribute vector, introduced in [11, is employed to find corresponding voxels in the ultrasound volumes. The elements of the attribute vector are revised to meet the requirements of ultrasound images. Using the attribute vectors, some leading points are automatically selected in the moving image, and their corresponding points in the fixed image are found. Finally, the moving image is warped into the fixed image.

This paper is organized as follows. Section 2 talks about the methodology and details of the implementation. Experimental results on 3D liver images are presented in Section 3 Section 4 concludes the paper and presents the future work.

\section{Method}

The proposed algorithm is demonstrated in Figure 1 At the first step, both fixed and moving images are passed through a Gaussian filter. This filter is used to generate more robust attribute vectors by reducing the effect of speckle, while providing different scales of images by adjusting its variance. In the next step, the elements of attribute vectors are calculated in two different scales for each voxel in both fixed and moving images. After this, the leading points with the highest magnitude attribute vectors are selected and registered, and finally the moving image is warped to the fixed image. The following paragraphs explain these steps in details.

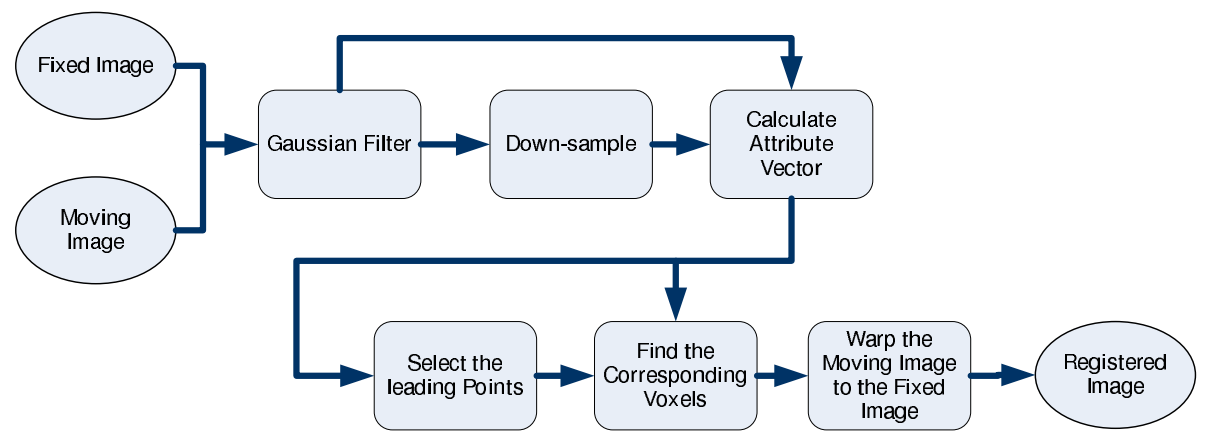

Fig. 1. The registration process

\subsection{Attribute Vectors}

Inspired by [12, we have used the concept of attribute vectors to extract corresponding feature points from the two ultrasound images. In [12] GMIs (geometric moment invariants) calculated from local spatial intensity histograms, form the attribute vector, which is assigned to each voxel in the image. Each attribute vector reflects the underlying anatomy and distinguishes between different features in an image.

Due to the presence of speckles in ultrasound images, our experiments show that GMIs do not generate robust attribute vectors, as they mainly characterize the speckle pattern. Depending on the properties of the imaging modality, 
other elements could be selected for the attribute vector. Our proposed method replaces GMIs with a set of new elements explained in the following paragraph to consider the properties of ultrasound images.

The first element of our attribute vector is the intensity of the voxel, which guarantees that the bright voxels will not be registered to the dark ones and vice versa. The other two elements are the magnitude of the gradient and the second derivative (Laplacian of the Gaussian), which are selected to extract the boundary information of the features in the images. All the elements are calculated in two scales. The scale with higher variance reveals the more general specifications, and the one with lower variance depicts the more detailed features. The generated attribute vector is able to robustly find the correspondence across the images taken from the same anatomy at different times. All the elements are normalized between zero and one in order to fit the similarity function, described later. The variance of each element could also be used in the normalization process. The next step is to select the leading points which is described in the following section.

\subsection{Leading Point Selection}

One of the major advantages of our proposed technique is its fast computational speed. To this end, instead of searching for corresponding voxel points for all the voxels within the moving and fixed image data sets, the algorithm automatically selects distinct major features of the moving image and registers them to the fixed image. The displacement of the rest of the voxel points are simply calculated based on the major feature points' displacements. This selection process includes the calculation of an importance function for each voxel. The importance of voxel $v$ is simply defined as:

$$
i m(v)=\sum_{i=1}^{n}\left(w_{i} \times e_{i}(v)\right)
$$

where $e_{i}(v)$ is the $i$ 'th element of attribute vector at point $v$, and $n$ is the number of elements of attribute vector. $w_{i}$ is the weight selected for $i$ 'th element. The points that their importance function is greater than a threshold are selected as leading points. Our experiments show that the number of leading points is not very sensitive to the selected threshold. These leading points are processed in a descending order based on the values assigned from Equation (11) to each of them. Once a leading point is selected, no more points within a certain radius to that point are selected in order to decrease the number of leading points, and hence, increase the speed of registration. In the current implementation the radius size is set to $1.2 \mathrm{~mm}$. This is done by setting the importance function around the selected point to zero, after its selection.

Registration of the selected points has lower risk of local minima, since they are usually located on the edge points in images. These boundary voxels have characteristics that are clearly distinguishable from neighbor areas using our proposed attribute vectors. 


\section{$2.3 \quad$ Matching}

Let $e_{i}(v)$ be the $i$ 'th element of attribute vector assigned to voxel $v$. Then, the similarity of two voxels $v_{1}$ and $v_{2}$ is measured with:

$$
\operatorname{Sim}\left(v_{1}, v_{2}\right)=\prod_{i=1}^{n}\left(1-\left\|e_{i}\left(v_{1}\right)-e_{i}\left(v_{2}\right)\right\|\right)^{w_{i}}
$$

where $\operatorname{Sim}\left(s_{1}, s_{2}\right)$ shows the similarity of attribute vectors at points $s_{1}$ and $s_{2}$, which is one for maximum similarity and zero for no similarity. $w_{i}$ depicts the contribution of $i$ 'th element of attribute vector in similarity function. If $w_{i}$ 's are all set to one, all the elements of the attribute vector will have the same contribution in determining the similarity.

To find the corresponding voxels in the fixed and moving images, the minimum of an energy function is found in a search area around the coordinates of the voxel in the fixed image. The energy function uses the described similarity function for several points around the two center points. The size of the search area is application dependent, and is directly proportional to the amount of deformation between the two data sets being registered. The advantage of using a limited search area is to reduce the computational complexity of the algorithm, as well as reducing the possibility of getting trapped in local minima. Our energy function is similar to but not exactly the same as the ones defined in [8, 11]: Let $p_{i}$ be the $i$ 'th voxel in the neighborhood of the center point $p$, and $q_{j}$ be the $j$ 'th voxel in the neighborhood of the center point $q$. The energy function, when examining the correspondence of $p$ and $q$, will be:

$$
\operatorname{Eng}(p, q)=\frac{\sum_{i} w(i)\left(1-\operatorname{Sim}\left(p_{i}, q_{i}\right)\right)}{\sum_{i} w(i)}+\alpha d(p, q)
$$

where $w_{i}$ is a weighting function which is maximum in the center of search area, $d(p, q)$ is the distance of $p$ from $q$, and $\alpha$ is a small coefficient. When the two sets of pixels $p_{i}$ 's and $q_{i}$ 's are similar, the energy function is minimized. The second term causes the energy function to be minimum at closer points to the center, when there is no absolute minimum. The reason for adding this term is that when there are two candidates for the corresponding voxel, the probability that the closer voxel is the right choice is higher.

Due to shadowing effects and the nature of ultrasound images, it is possible that a feature disappears from one image to the other. To face this phenomenon, when a match is found, the energy function at the matching point should be less than a threshold. Otherwise, that point is removed from the list of leading points. In all of our experiments for different data sets and deformations in the volumes, a single threshold value was used.

\section{$2.4 \quad$ Warping}

The final step of the registration process is to calculate the movement of all the voxels of the moving image with respect to the fixed image, based on the 
calculated displacement of the leading feature points. The displacement of each voxel is mainly determined by the leading voxels that are close to that point. Displacement of each leading voxel is distributed to the points around it by a Gaussian function, which means that the displacement is faded through the neighbors. The variance of the function is set according to the volume scale factors. The faded displacement vectors of leading points could not be simply added together. Otherwise, since the selected leading voxels might be anywhere in the image, the displacement of the points between two corresponding leading voxels in the moving and the fixed images might get much larger than the original displacement. Therefore, the leading voxels contribute to the displacement of each point, inversely proportional to their distance to the point. The following equation is used in the warping process for voxel $v$ :

$$
\operatorname{Disp}(v)=\frac{\sum_{i=1}^{n_{\text {lead }}} f_{v}\left(d_{i}\right) G\left(d_{i}\right) \operatorname{Disp}\left(l_{i}\right)}{\sum_{i=1}^{n_{\text {lead }}} f_{v}\left(d_{i}\right)}
$$

where $l_{i}$ 's are the leading voxels, and $n_{\text {lead }}$ is the number of them. $d_{i}$ is the distance of $v$ to $l_{i}$, and $f_{v}\left(d_{i}\right)$ is the inverse proportional function defined as $\frac{1}{d_{i}^{2}+\epsilon}$, where $\epsilon$ is a small positive number. $G$ is the Gaussian function that fades the effect of far leading voxels. The inverse proportional function causes the leading points to be warped exactly to their corresponding voxels, found in previous step.

\section{Results}

Ultrasound images were captured by a GE Voluson 730 3D/4D ultrasound machine. The RAB4-8P transducer was used to collect 3D images from two volunteers' livers, and the images were saved in Cartesian format. These images were captured at multiple locations under different forces applied to the abdomen by various operator's hand pressures on the transducer. The deformation in ultrasound volume data sets was also caused by respiration, heartbeat and changes in the physical position of the volunteers. For each volunteer, five sets of images were captured. Each set of images, consisted of two volumes at two different moments of the breathing cycle, were taken from one part of the volunteer's liver. For each set, one of the volumes was selected as the fixed image, and the other one (moving image) was registered to it. The average displacement between the images in each set was estimated to be 8.7 voxels with a maximum displacement of 20.8 voxels (the scale factor was set to $0.6 \mathrm{~mm} /$ voxel).

The code has been implemented using MATLAB ${ }^{\mathrm{TM}}$. The matching and warping parts are written in $\mathrm{C}$, and compiled with the $\mathrm{C}$ compiler of MATLAB ${ }^{\mathrm{TM}}$ to reduce the computation time. Figures $2(a, b, c)$ demonstrate one slice of the fixed, moving and registered data sets. The difference images shown in Figures 2 (d,e) depict the accuracy of the alignment of features after registration. It should be noted that the registration is performed in $3 \mathrm{D}$, therefore some out-of-plane features, which may not exist in the demonstrated moving and fixed images, 


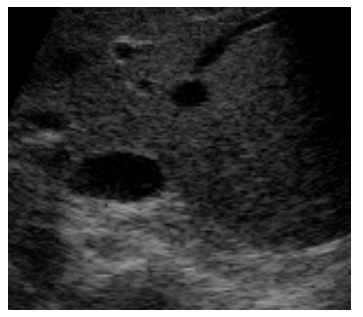

(a)

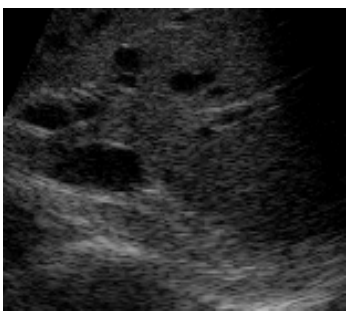

(b)

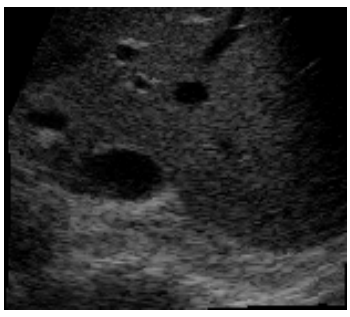

(c)

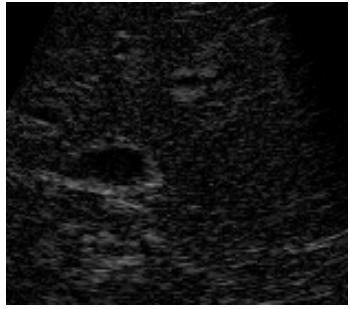

(d)

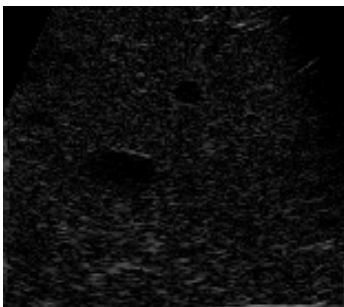

(e)

Fig. 2. (a) is a middle slice of the fixed volume, (b) is the same slice in the moving volume, and (c) is the same slice in the registered volume. (d) shows the absolute of difference between the fixed and the moving volumes, and (e) is the absolute of difference between the fixed and the registered volumes.

have been warped to the shown registered slice. Figure 3 shows the results of another experiment. Three slices in three orthogonal planes are demonstrated. The experiments show that the algorithm is robust, accurate and not sensitive to local minima, when the maximum displacement caused by deformation is less than the radius of the search area.

To evaluate the accuracy of the technique, two experiments were carried out. First, eight voxels on distinct features of the fixed and the registered volumes were manually selected. The distance between the corresponding voxels were then considered as the registration error. Using this method, the average error of registered images was about two voxels on average considering the uncertainties in the images and poor quality of them. In the second experiment, the matrices of displacement vectors generated in the previous experiments were used to simulate natural deformation. The original volumes were then registered to the deformed volumes, and the results were compared to the original displacement matrices. The average of mean error for 20 volumes deformed with this method was 1.50 voxels.

For two 185x113x199 voxels volumes, the program took about 436 seconds to finalize registration on a Pentium $42.4 \mathrm{GHz}$ with $512 \mathrm{MB}$ RAM machine. Pre-filtering and calculating the attribute vectors took about 74 seconds; The time required for selection of leading points and registering them was about 203 seconds, and warping took the rest of time. 


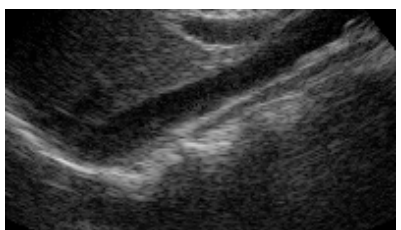

(a) fixed

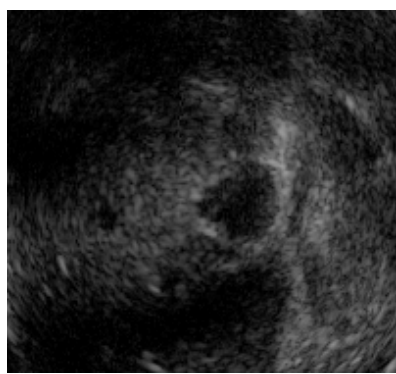

(d) fixed

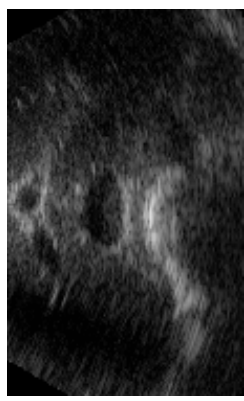

(g) fixed

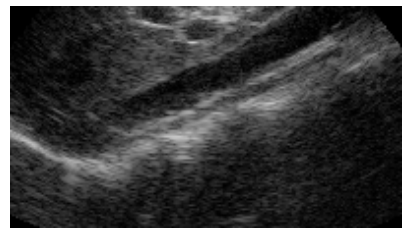

(b) moving

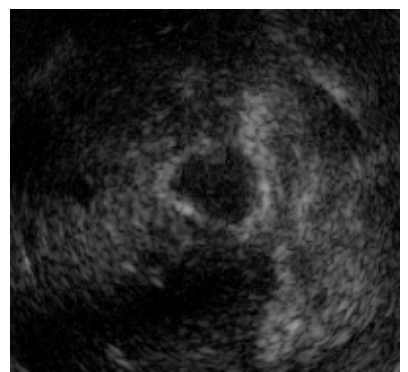

(e) moving

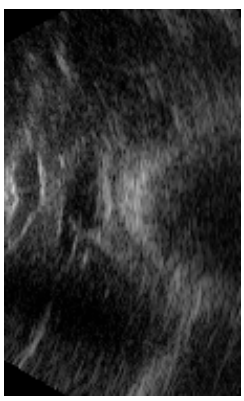

(h) moving

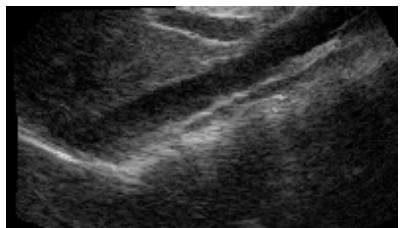

(c) registered

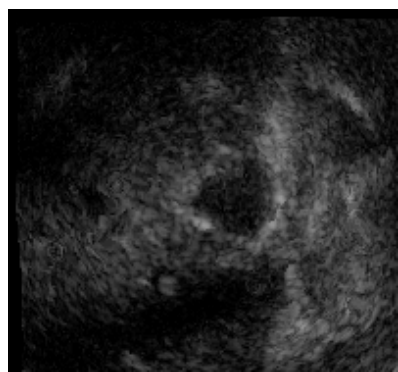

(f) registered

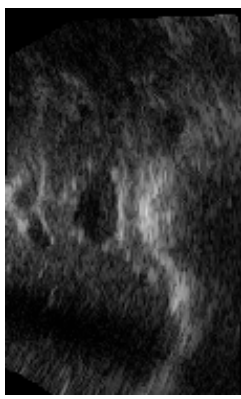

(i) registered

Fig. 3. (a),(b), and (c) are the slices of fixed, moving, and registered volumes, parallel to ZY plane; (d) to (f) are the slices parallel to ZX plane, and (g) to (i) are the slices parallel to XY plane

\section{Conclusion and Future Work}

We have developed a new method for 3D elastic registration of ultrasound images, which is robust and flexible and is intended eventually for intra-operative surgical applications. The algorithm does not require any pre-segmentation or numerical optimization, and as a result, the running time is decreased. There are a number of parameters, such as the size of the search area, that should be set correctly for the algorithm to function properly. However, as long as the search area size is larger than the deformation between the two image data sets, the performance of the technique is not very sensitive to the changes in these 
parameters. Future work will include the optimization of the code to reduce the running time of the algorithm to within an acceptable range for intra-operative applications, as well as improving the normalization method of the elements of the attribute vector. The algorithm should also be tested for the registration of ultrasound images of other anatomical parts in addition to the liver.

\section{References}

1. Aylward, S.R., Jomier, J., Guyon, J.P., Weeks, S.: Intra-operative 3d ultrasound augmentation. In: IEEE International Symposium on Biomedical Imaging. (2002) 421-424

2. Pagoulatos, N., Edwards, W.S., Haynor, D.R., Kim, Y.: Interactive 3d registration of ultrasound and magnetic resonance images based on a magnetic position sensor. IEEE Transactions on Information Technology in Biomedicine 3 (1999) 278-288

3. King, A.P., Blackall, J.M., Penney, G.P., Hawkes, D.J.: Tracking liver motion using 3-d ultrasound and a surface based statistical shape model. In: Mathematical Methods in Biomedical Image Analysis. (2001) 145-152

4. Arbel, T., Morandi, X., Comeau, R.M., Collins, D.L.: Automatic nonlinear mriultrasound registration for the correction of intra-operative brain deformations. In: MICCAI. (2001) 565572

5. King, A.P., Blackall, J.M., Penney, G.P., Edwards, P.J., Hill, D.L.G., Hawkes, D.J.: Baysian estimation of intra-operative deformation for image-guided surgery using 3-d ultrasound. In: MICCAI. (2000) 588597

6. Shekhar, R., Zagrodsky, V.: Mutual information-based rigid and nonrigid registration of ultrasound volumes. IEEE Transactions on Medical Imaging 21 (2002) 9-22

7. Krucker, J.F., LeCarpentier, G.L., Fowlkes, J.B., Carson, P.L.: Rapid elastic image registration for 3-d ultrasound. IEEE Transactions on Medical Imaging 21 (2002) $1384-1394$

8. Xiao, G., Brady, M., Noble, J.A., Burcher, M., English, R.: Nonrigid registration of 3-d free-hand ultrasound images of the breast. IEEE Transactions on Medical Imaging 21 (2002) 405-412

9. Shekhar, R., Zagrodsky, V., Garcia, M.J., Thomas, J.D.: Registration of real-time 3 -d ultrasound images of the heart for novel 3-d stress echocardiography. IEEE Transactions on Medical Imaging 23 (2004) 1141-1149

10. Makela, T., Clarysse, P., Sipila, O., Pauna, N., Pham, Q.C., Katila, T., Magnin, I.E.: A review of cardiac image registration methods. IEEE Transactions on Medical Imaging 21 (2002) 1011-1021

11. Shen, D., Davatzikos, C.: Hammer: hierarchical attribute matching mechanism for elastic registration. IEEE Transactions on Medical Imaging 21 (2002) 1421-1439

12. Shen, D.: Image registration by hierarchical matching of local spatial intensity histograms. In: MICCAI. (2004) 582-590 